Results 34 EMRs were performed in nine patients requiring anticoagulation. 8 were on warfarin due to atrial fibrillation, one took warfarin after pulmonary embolism. One patient on warfarin was readmitted 10 days after EMR with haematemesis, melaena and an drop in haemoglobin $>5 \mathrm{~g} / \mathrm{dl}$ caused by bleeding from an EMR resection ulcer; the bleeding had settled spontaneously at the time of endoscopy. Out of 138 EMRs in 35 controls, five acute bleeding events occurred during EMR which required treatment by clipping, coagulation grasper or heater probe. No delayed bleeding event occurred in the control group. The maximal diameter of the resected specimen did not differ between anticoagulated patients and controls (median $17 \mathrm{~mm}$; 25\%>75\% percentile: $15-18 \mathrm{~mm}$ vs $17 \mathrm{~mm}$; 25\%>75\% percentile: $15-20 \mathrm{~mm}$; $\mathrm{p}=0.68)$. No perforations were observed in either groups. The number of bleeding events did not differ between groups $(p=0.85)$, neither for acute $(p=0.60)$ or delayed bleeding $(p=0.46)$.

Conclusion EMR of early oesophageal neoplasia can be safely performed in patients requiring anticoagulation when warfarin is discontinued 5 days before the endoscopic intervention and reinstituted on the evening of the procedure day.

Competing interests None declared.

\section{PWE-215 NUMERICAL ANALYSIS OF COLOUR FLUORESCENCE RATIO AND CONTRAST USING VIDEO AUTOFLUORESENCE ENDOSCOPY ACCURATELY IDENTIFIES HIGH GRADE DYSPLASIA OR CANCER IN PATIENTS WITH BARRETT'S OESOPHAGUS}

doi:10.1136/gutjnl-2012-302514d.215

\begin{abstract}
1,2V Subramanian, * ${ }^{3} \mathrm{~J}$ Mannath, ${ }^{4} \mathrm{D}$ F Boerwinkel, ${ }^{4} \mathrm{~L}$ Alvarez-Herrero, ${ }^{4} \mathrm{~W}$ L Curvers, ${ }^{3} \mathrm{C} \mathrm{J}$ Hawkey, ${ }^{4} \mathrm{~J} \mathrm{~J}$ Bergman, ${ }^{3} \mathrm{~K}$ Ragunath. ${ }^{1}$ Department of Gastroenterology, Leeds Teaching Hospitals NHS trust, Leeds, UK; ${ }^{2}$ Leeds Institute of Molecular Medicine, St James University Hospital, Leeds, UK; ${ }^{3}$ Nottingham Digestive Diseases Centre, Nottingham University Hospital, Nottingham, UK; ${ }^{4}$ Department of Gastroenterology and Hepatology, Academic Medical Centre, Amsterdam, Netherlands
\end{abstract}

Introduction Autofluorescence endoscopy (AFE) is a novel technique that identifies early neoplasia in Barrett's oesophagus (BE) by highlighting differences in tissue autofluoresence (AF). It has high sensitivity but is associated with false positive rates up to $80 \%$. We aimed to develop numerical measures of AF to reduce the false positive rates of AFE.

Methods Images of AFE lesions in patients with BE were prospectively collected. Blinded anonymised images were de-gamma corrected and average grayscale values in the red, green and blue channels of the abnormal and background normal area were quantified. The autofluoresence intensity (ratio of average red to green channel greyscale value of lesion compared to background), colour contrast index (between lesion and background), hue, saturation and lightness (of the lesion) were calculated. A decision tree based on the training set was developed with the J48 algorithm in WEKA3.2.4, using a 10-fold cross validation strategy. The performance of the model developed was assessed on an independently collected test dataset.

Results There were 82 images (37 high grade dysplasia/cancer) in the training set and 164 images (51 high grade dysplasia/cancer) in the test set. The decision tree classifier developed utilised only autofluoresence intensity and colour contrast index and had a sensitivity of $97 \%$, specificity of $77 \%$, and negative predictive value of $98 \%$ in detecting high grade dysplasia/cancer in the independent test set. The false positive rate of AFE was reduced from $70 \%$ to $16 \%$.

Conclusion Numerical analysis of colour fluorescence and contrast is a reliable, objective and accurate method of reducing the false positive rate of AFE and can be easily incorporated into real time endoscopy.
Competing interests V Subramanian: None declared, J Mannath: None declared, D Boerwinkel: None declared, L Alvarez-Herrero: None declared, W Curvers: None declared, C Hawkey: None declared, J Bergman Grant/Research Support from: Olympus Medical, K Ragunath Grant/Research Support from: Olympus Medical.

\section{PWE-216 DIAGNOSTIC ACCURACY FOR DETECTION OF DYSPLASIA IN BARRETT'S OESOPHAGUS USING NARROW BAND IMAGING WITH MAGNIFICATION IMPROVES AFTER A SHORT TRAINING SESSION IN THOSE WITH PRIOR EXPOSURE TO NBI BUT NOT IN NOVICES}

doi:10.1136/gutjnl-2012-302514d.216

1,2V Subramanian, * ${ }^{3} \mathrm{~K}$ Ragunath. ${ }^{1}$ Department of Gastroenterology, Leeds Teaching Hospitals NHS trust, UK; ${ }^{2}$ Leeds Institute of Molecular Medicine, St James University Hospital, Leeds, UK; ${ }^{3}$ Nottingham Digestive Diseases Centre, Nottingham University Hospital, Nottingham, UK

Introduction Narrow band imaging (NBI) with magnification (NBIzoom) has been shown to be highly sensitive and specific in identifying dysplasia in Barrett's oeosphagus (BO). These studies have been done using experts and there is limited data on how these techniques can be taught. Recently studies have demonstrated that identifying adenomatous pit patterns in colonic polyps using NBI can be easily taught by short training sessions. The aim of this study was to prospectively evaluate the utility of a short training session on the ability of endoscopists to identify dysplastic patterns in NBIzoom images from patients with $\mathrm{BO}$.

Methods A prospective observational study was done on 28 endoscopists (10 novices, 10 with prior exposure to NBI and 10 experienced in the use of NBI-zoom) who attended a national level symposium on BO. A pre-test with $15 \mathrm{NBI-zoom} \mathrm{images} \mathrm{was}$ followed by a 15 min training session where the endoscopic criteria for making a diagnosis of dysplasia on NBI-zoom images were described and demonstrated. The post-test had 25 NBI-zoom images. Images were projected using a high definition projector system and participants were given $45 \mathrm{~s}$ per image to make a diagnosis. Overall accuracy in detecting dysplasia was calculated and the inter-observer reliability of the groups was determined using bias corrected $\kappa$ statistics.

Results Abstract PWE-216 table 1 provides the accuracy of classification of mucosal and vascular patterns and identifying the presence of dysplasia in the three groups of endoscopists. Significant improvement was noted after the training session in the group with prior NBI exposure but not in novices. The $\kappa$ value for inter-observer reliability significantly $(p=0.01)$ improved among those with prior NBI exposure from $0.238(0.082-0.453)$ to $0.650(0.421-0.836)$ after the training sessions but not in novices $(p=0.4) 0.280(0.084-0.532)$ to $0.374(0.251-0.517)$ or experienced endoscopists $(\mathrm{p}=0.08) 0.505$ (0.273-0.708) to $0.779(0.552-0.929)$.

\section{Abstract PWE-216 Table}

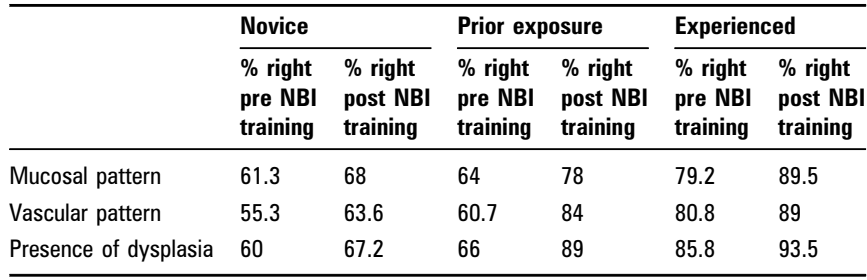

Conclusion A short didiactic teaching session can achieve high accuracy and good interobsever agreement in identifying dysplastic patterns in NBI-zoom images from $\mathrm{BO}$ in those with some prior NBI experience but not in novices.

Competing interests V Subramanian: None declared, K Ragunath Grant/Research Support from: Olympus Medical. 